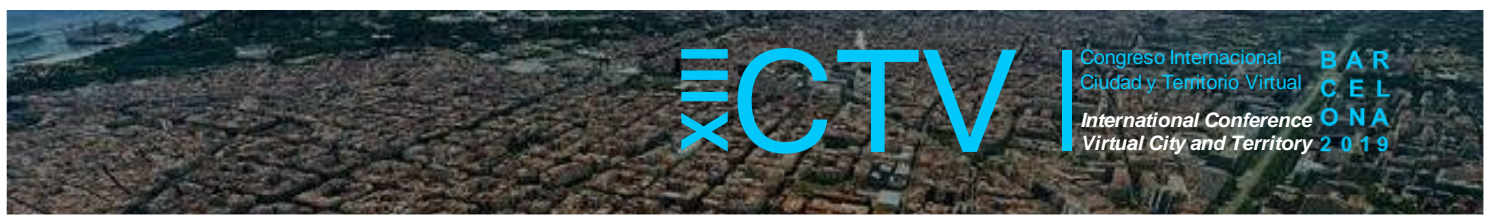

\title{
ESTUDIO COMPARATIVO DE LA ECOEFICIENCIA URBANA. CASO DE ESTUDIO: DISTRITO SARRIÀ - SANT GERVASI
}

\author{
Sepúlveda Navarro, Leonardo ${ }^{1 *}$; Roca Cladera, Josep ${ }^{2}$ \\ Remisión inicial: 2019-06-16; Remisión definitiva: 2019-10-12; Publicación: 2019-12-21
}

Citación: Sepúlveda, L y Roca, J. et al. (2019). Estudio comparativo de la ecoeficiencia urbana. Caso de estudio: distrito Sarrià - Sant Gervasi. En XIII CTV 2019 Proceedings: XIII International Conference on Virtual City and Territory: "Challenges and paradigms of the contemporary city": UPC, Barcelona, October 2-4, 2019. Barcelona: CPSV, 2019, p. 8662. E-ISSN 2604-6512. DOI http://dx.doi.org/10.5821/ctv.8662

\section{Resumen}

Basado en los estudios referidos a la ciudad como estructura disipativa y la teoría de la información aplicada al ámbito urbano, la presente investigación se enfoca en el análisis de la entropía física (S), como un indicador del grado de deterioro ambiental, generado por el metabolismo del sistema urbano y su relación con la producción de entropía negativa o neguentropía $(H)$ que compensa el impacto negativo sobre el medio ambiente. Este tipo de entropía se relacionó con el teorema de Shannon, el cual permite medir la cantidad de información generada por un sistema, en este caso los barrios a estudiar. La información, expresada en bits, refleja el grado de complejidad de la ciudad, la cual se asocia en general, a los atributos positivos de los modelos de ciudad compacta y diversa.

La principal problemática referida a este tipo de evaluación es la falta de información desagregada a escalas inferiores a las de las ciudades (distritos y barrios), principalmente respecto a los indicadores de entropía física tales como: consumo energético, producción de desechos, entre otros.

El objetivo del estudio es desarrollar un ejemplo de metodología de evaluación ambiental comparativa, que permita cualificar de forma simplificada un ámbito urbano especifico, a través de una ratio aplicable a distintas escalas.

Este estudio comparativo se centra en el análisis del distrito de Sarriá - Sant Gervasi de la ciudad de Barcelona y los 6 barrios que lo componen, en el que se calcularon separadamente las variables entropía física (S) y la variable entropía negativa $(\mathrm{H})$. La variable $\mathrm{S}$, se simplificó solo al cálculo del consumo energético por barrio y su correspondencia en emisiones de CO2 y la variable $S$ fue calculada aplicando la fórmula de Shannon a 4 indicadores urbanos relacionados con la complejidad y diversidad de cada barrio. Para el cálculo de $S$ se analizaron los datos de los consumos energéticos y su correspondencia en emisiones de $\mathrm{CO} 2$ a nivel de distritos, presentes en el documento de Balance energético de Barcelona, disponible en la página web del observatorio de energía, del departamento de ecología, urbanismo y movilidad del ayuntamiento de Barcelona. A partir de la comprobación de una serie de correlaciones entre el consumo energético total distrital e índices tales como el de renta per cápita bruta y las superficies catastrales a escala de ciudad, se generaron una serie de modelos de regresión lineal, a través del programa IBM SPSS STATICS, con los cuales se desagregan los datos de consumo y emisiones de $\mathrm{CO} 2$ a cada barrio del distrito estudiado.

En cuanto al índice $\mathrm{H}$, se calculó con la fórmula de la entropía Shannon, aplicada a los datos obtenidos principalmente desde la página web del departamento de estadísticas y datos del ayuntamiento de Barcelona, que tienen relación con la diversidad de cada barrio: Inventario de locales comerciales en $1^{\circ}$ piso, locales catastrales según uso, nivel académico de la población, nacionalidad de la población.

Finalmente, para calcular el índice de ecoeficiencia se aplica la formula H/S, la cual divide los resultados de la producción anual de $\mathrm{CO} 2$ por barrio, por el promedio ponderado de los cálculos de complejidad urbana para cada uno los 4 índices analizados.

\footnotetext{
${ }^{1}$ Arquitecto, jefe de taller, oficina AURA LTDA., Santiago de Chile, Master en Planificación urbana y sostenibilidad, UPC, https://orcid.org/0000-0002-9247-7003; ${ }^{2}$ Dr. Arquitecto, Catedrático del Departamento de Tecnología de la Arquitectura UPC, https://orcid.org/0000-0003-3970-6505. * Correo de contacto: Isepulvedarq@gmail.com
} 


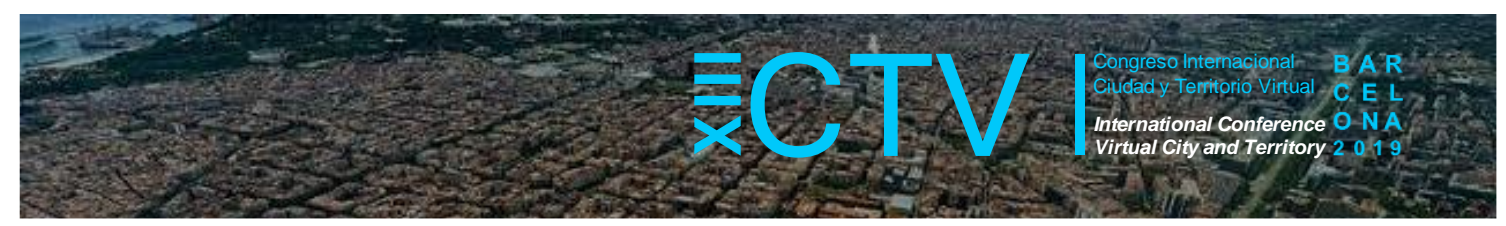

Los resultados principales se pueden expresar en lo siguiente: el barrio de Sant Gervasi - Galvany obtuvo un índice $S$ de 1.32 tCO2eq, un índice $\mathrm{H}$ de 2.127 bits y un índice de ecoeficiencia de $1.606 \mathrm{tCO} e q / \mathrm{bits}$. En cuanto al barrio de Sarriá, este obtuvo un índice $\mathrm{S}$ de 0.81 tCO2eq, una magnitud H de 2.256 bits y un índice de ecoeficiencia de 2.798 tCO2eq/bits.

De los resultados anteriores podemos inferir que: El barrio que más entropía física obtuvo, Sant Gervasi - Galvany, es el menos ecoeficiente, la información que produce no compensa las altas emisiones de $\mathrm{CO} 2$ que el sector comercial y de servicios genera. Por otro lado, el barrio de Sarrià es el que menos genera entropía física y a su vez es el segundo barrio más diverso del distrito.

Palabras Clave: Ecoeficiencia; Entropia; Neguentropia

\section{Introducción: La ciudad como un sistema disipativo}

La idea del impacto global respecto a las consecuencias medioambientales del desarrollo económico de las Naciones ha tomado especial relevancia en las últimas décadas, sobre todo desde la perspectiva del fenómeno del calentamiento global, que a la fecha ya es un proceso indiscutible. (UN, 2000).

Por otro lado, en la actualidad más de la mitad de la población mundial vive en ciudades ${ }^{2}$. Esta población a pesar de que ocupa alrededor de un $3 \%$ de la superficie terrestre consume entre un $60 \%$ y un $80 \%$ del total de energía global, contribuyendo al $75 \%$ de las emisiones de carbono, las que representan un $30 \%$ anual de las emisiones de gases efecto invernadero (IPCC, 2014).

El debate sobre la influencia negativa de las ciudades sobre el medio ambiente y la necesidad de enfocar la creciente expansión urbana global ${ }^{3}$, desde una perspectiva de desarrollo sostenible, ha generado la necesidad de contar con herramientas que permitan un análisis y cuantificación objetiva y replicable, del grado de influencia de las ciudades, sobre el medio natural.

Una forma de abordar esta problemática se deriva de la interpretación del medio urbano como un sistema disipativo. Esta definición se extrapola de las ciencias naturales, desde el concepto de "estructuras disipativas", desarrollado por el autor llya Priogine, premio Nobel de Química, el cual las define como sistemas complejos, abiertos y autoorganizados, que no poseen equilibrio interno, pero que tienden al Orden y la disipación en cuanto a su relación con la energía y la materia. "Las características generales de estas estructuras ordenadas son esencialmente las siguientes: se desarrollan muy lejos del equilibrio, es decir, son fenómenos fuertemente irreversibles $y$, por tanto, fuertemente disipativos (de energía o materia). A causa de esta fuerte disipación, que tienen que compensar para poder mantenerse, estas estructuras sólo aparecen en sistemas que intercambian materia y/o energía con su entorno, es decir, en sistemas abiertos" (García, 1980).

Así, en este enfoque, la ciudad se podría entender como una estructura compleja, con una autoorganización sin un orden interno intrínseco que, por principio, en su condición de

\footnotetext{
${ }^{2}$ El año 2018 el 54\% de la población mundial vive en áreas urbanas. Datos de banco mundial. (Banco Mundial).

${ }^{3}$ Se estima que más del $70 \%$ de las emisiones de CO2 son producidas en el medio urbano. (IPCC, 2018)
} 


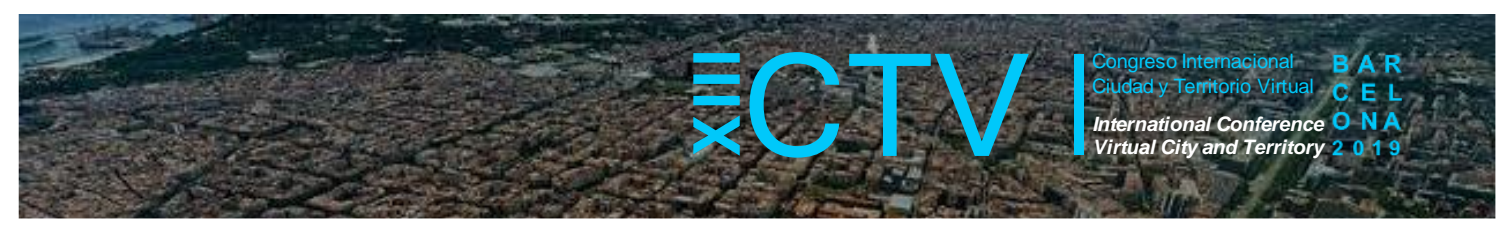

disipador de energía, tiende a aumentar la entropía asociada la transformación irreversible de la materia y energía.

\subsection{Metabolismo Urbano y la entropía física}

Uno de los enfoques respecto al tema es desarrollado, desde el trabajo pionero de Wolman (1965), el cual define el análisis del metabolismo urbano (MU) como "Una plataforma multidisciplinaria e integrada que analiza los flujos de materiales y energía en las ciudades, entendidas como sistemas complejos que son condicionadas por variados factores sociales, económicos y medioambientales. Similarmente a los organismos biológicos y los ecosistemas, los ciclos urbanos y sus transformaciones, requieren materiales, comida, agua y combustible para generar estructuras físicas, biomasa y desechos." (Wolman, 1965).

En cuanto al estado del arte de este enfoque, existen dos aproximaciones al cálculo y comprensión del $M U$ :

- La aproximación MEFA (Mass Energy Flow Análysis), con sus distintas variantes, basadas en la contabilización de unidades convencionales de energía y masa.

- La aproximación ecológica, basada en el trabajo sobre la Emergía (Odum, 1983). La Emergía se define simplificadamente como la exergía de un determinado tipo, que se ha usado directa o indirectamente en las transformaciones necesarias para generar un producto o servicio. Uno de los conceptos claves de este enfoque es el reconocimiento de la diferenciación de las distintas "Calidades" de energía, la cual es generada primariamente por la Energía solar, por lo que todo debe medirse en términos de "emergía" solar.

Ambas posturas conllevan implícitamente, el concepto de entropía como un fenómeno intrínseco a la ciudad, entendiendo esta como una magnitud que puede expresar el grado de eficiencia respecto al uso de la energía y la materia. Desde el trabajo de Boltzmann (1909) que relacionó la entropía térmica con la entropía estadística, podemos relacionar este término con concepto de "orden" definido por Peter Landsberg como "La entropía existente dividida por la entropía máxima alcanzable", la anterior formula establece una relación directamente proporcional entre desorden y entropía. Esta idea de desorden se entiende mas bien como la desconfiguración de una estructura compleja a un estado de menor complejidad y mayor homogeneidad de sus partes (Fariña, 2002).

\subsection{Neguentropía y la entropía informativa}

La idea de irreversibilidad y aumento progresivo de la entropía en sistemas cerrados tiene una respuesta en el concepto de Neguentropía o entropía negativa (Schödinger, 1943), la cual puede entenderse como el mecanismo de los sistemas complejos de mantener una estabilidad u orden mitigando la progresión de la entropía. Esta mitigación, en el caso urbano, solo podría realizarse exportando entropía en forma de calor y desechos "Huella ecológica"; o ampliando la entropía máxima posible (ampliando el límite urbano, en el caso de las ciudades). Esta visión un tanto pesimista, tiene su contraparte desde la teoría de la información (Shannon), la cual relaciona directamente entropía e información. La información es definida como el grado de incertidumbre de un mensaje o la cantidad de información en bits que esta expresa. Esta producción de información se asocia al concepto de "Complejidad urbana", tema ligado a la 


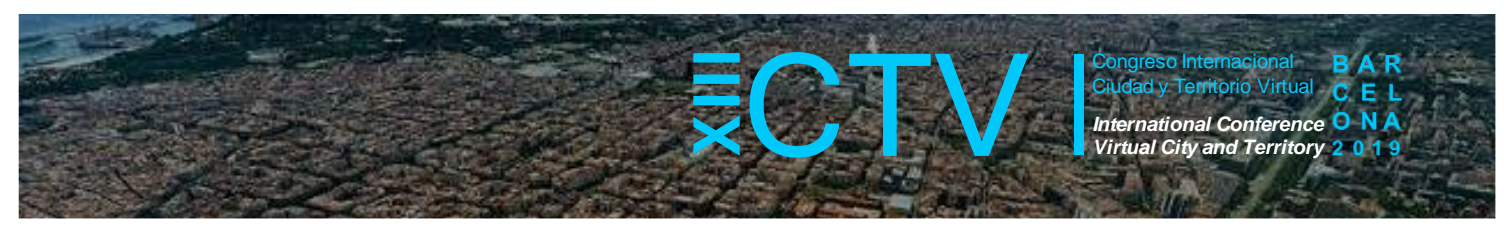

diversidad ecológica y que en general, se relaciona con los valores positivos relacionados a los modelos de ciudad compacta (Molini \& Salgado, 2010).

\subsection{Cálculo de la ecoeficiencia}

El concepto de ecoeficiencia tiene su origen en Consejo Empresarial Mundial para el Desarrollo Sostenible (WBCSD) de 1992, en donde la ecoeficiencia se define como "La ratio entre el valor añadido de lo que se ha producido y el impacto ambiental añadido que ha costado producirlo".

El anterior concepto aplicado al ámbito urbano, se puede definir como la relación entre la producción de entropía física, que representa en que grado ciudad como sistema genera un daño al medioambiente dividido por la entropía de la información, que representara el valor agregado de lo que produce la ciudad, en este caso la complejidad de su estructura expresada en bits de información.

\section{Objetivos}

El objetivo primario del estudio es desarrollar un ejemplo de metodología de evaluación ambiental comparativa, que permita cualificar de forma simplificada un ámbito urbano especifico, a través de una ratio aplicable a distintas escalas. La reducción de las variables que expresen las distintas entropías, a magnitudes de una relativa facilidad de obtención, es el principal interés de este trabajo, tomando en cuenta la endémica falta de monitorización del consumo energético y la producción de contaminantes en las ciudades. Como objetivo secundario el estudio busca encontrar los factores que inciden en la producción de las entropías para el ámbito de estudio.

\section{Metodología}

El presente estudio, desarrolla el cálculo de la ecoeficiencia y su comparación, para los 6 barrios que componen el distrito de Sant Gervasi - Galvany, ubicado al noroeste de la ciudad de Barcelona. Para la elección del caso de estudio, se tomo en cuenta que el distrito en cuestión es el que presenta el mayor índice de renta bruta familiar per cápita (RDF). Tomando como base la idea general que las comunas de mayores ingresos tienen los mayores consumos, lo que no se cumple en este caso, es de interés analizar los reales factores que inciden en los consumos totales de $\mathrm{CO} 2$ dentro de este ayuntamiento.

La metodología propone el cálculo del consumo energético y su correspondencia a consumo de $\mathrm{CO} 2$ como una forma simplificada de constatación de la entropía física que genera en cada barrio. La elección de la producción de $\mathrm{CO} 2$ como indicador único, se fundamenta en la relación de esta con parámetros del tipo huella ecológica ${ }^{4}$, que permite una comprensión intuitiva de la magnitud de daño ambiental producida, en este caso, por un ámbito urbano definido.

\footnotetext{
${ }^{4}$ La huella ecológica es un término desarrollado por William Rees y Mathis Wackernagel (1995), los cuales la definen como "el área de territorio productivo o ecosistema acuático necesario para producir los recursos utilizados y para asimilar los residuos producidos por una población definida con un nivel de vida específico, donde sea que se encuentre esta área". Esta magnitud también se entiende como el territorio necesario para la absorción del CO2 producido.
} 


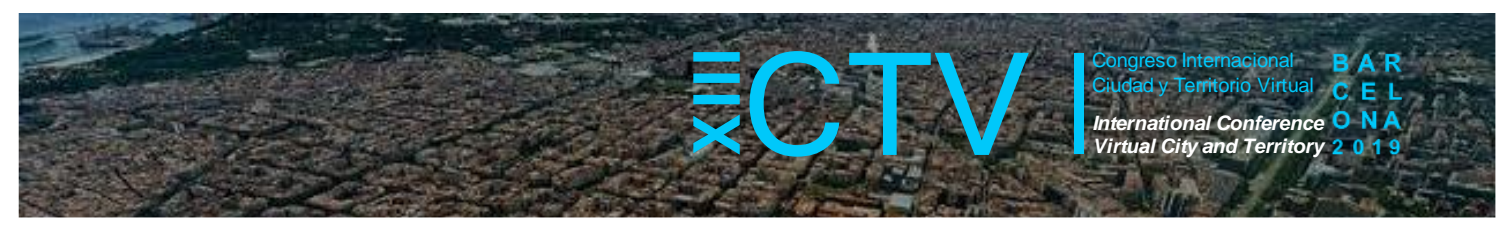

Figuras 1. Índice de renta bruta familiar y Consumo de CO2 per cápita, por distritos de Barcelona
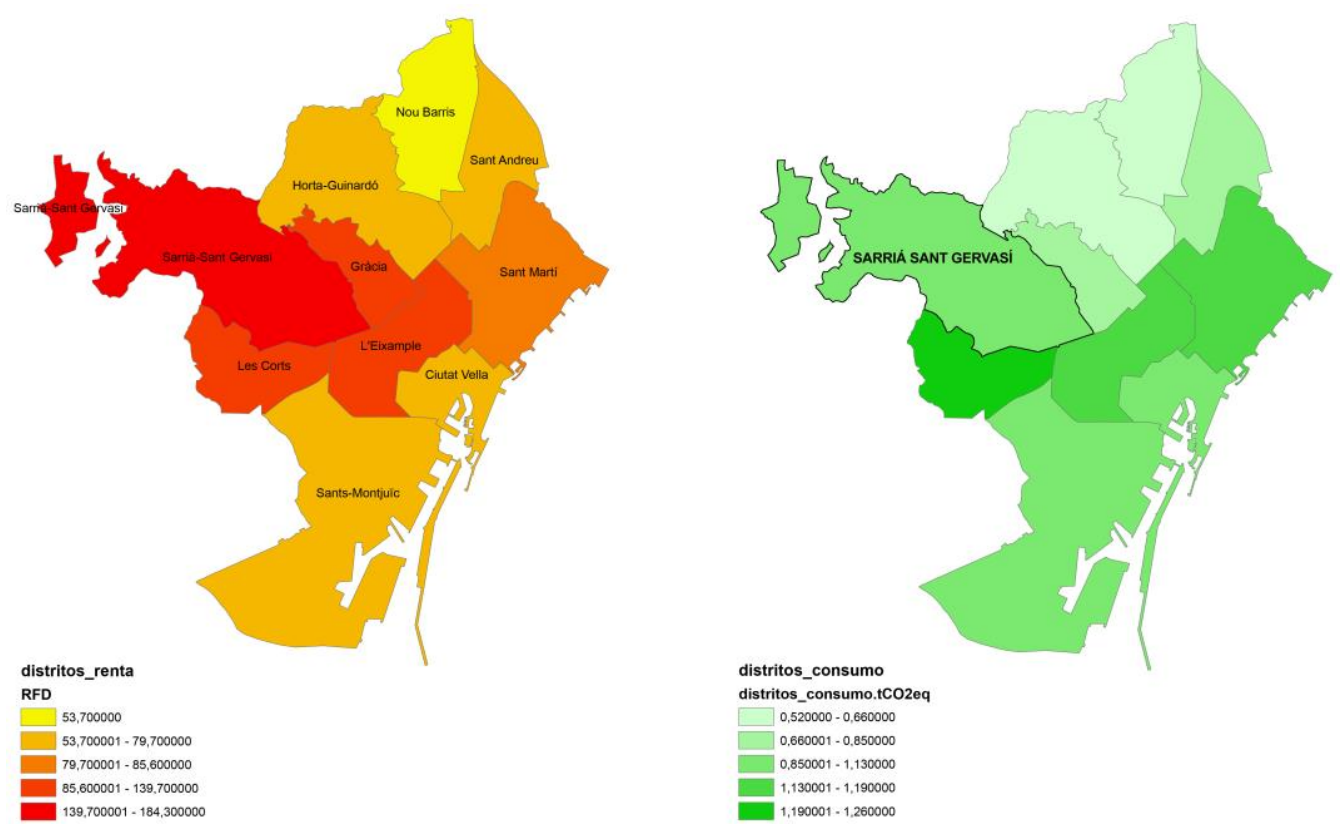

Fuente: Elaboración propia con datos del Ayuntamiento de Barcelona. (2014).

\section{Desarrollo}

\subsection{Cálculo de la entropía física}

El cuadro "Consum i emissions per districte lany 2014", del documento "Balance de la energía 2014", es el que presenta algunas cifras distribuidas a nivel de distrito. La tabla 1 contempla datos a nivel distrito, sobre el consumo doméstico, el de comercio - servicios y el industrial, excluyendo el transporte.

Tabla 1. Consumo y emisiones por distritos de Barcelona (2014)

\begin{tabular}{lcc}
\hline Distrito & MWh/hab. & tCOeq/hab \\
\hline Ciutat vella & 8.89 & 1.13 \\
\hline Eixample & 9.15 & 1.19 \\
\hline Sants-Montjuic & 8.18 & 1.12 \\
\hline Les Corts & 9.52 & 1.26 \\
\hline Sarriá-Sant Gervasi & 8.06 & 1.10 \\
\hline Gracia & 6.01 & 0.83 \\
\hline Hota-Guinardó & 4.69 & 0.66 \\
\hline Nou Barris & 3.72 & 0.52 \\
\hline Sant Andreu & 6.10 & 0.85 \\
\hline Sant Martí & 8.08 & 1.16 \\
\hline
\end{tabular}

Fuente: Elaboración propia desde datos del departamento de estadística y difusión de datos del Ayuntamiento de Barcelona. (2014). El documento "Balance de la Energía", especifica que los datos distribuyen el consumo de áreas como la industria o el comercio por cada habitante del término administrativo.

Comparando la distribución del consumo de energía y las superficies según usos catastrales, podríamos decir de esta relación que, el $15.53 \%$ del total construido en Barcelona, correspondiente al comercio y servicios, produce el $45 \%$ de todo el consumo de energía. En 


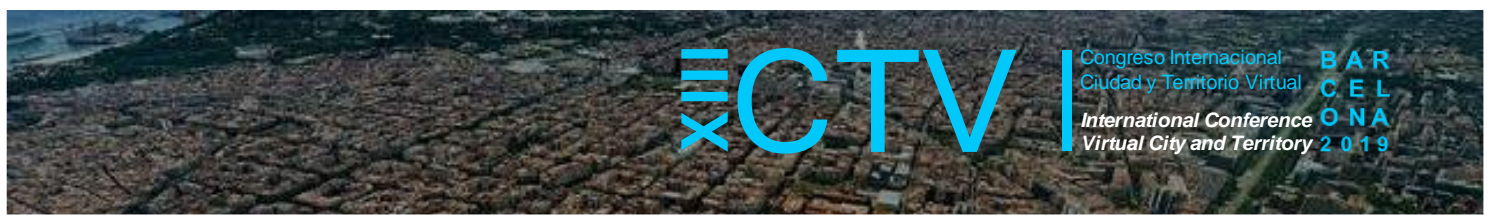

cuanto al uso doméstico el $56,27 \%$ de la superficie es el responsable solo del $37,78 \%$ del consumo total anual. Por otro lado, es razonable deducir una relación directa entre superficie construida y consumo energético para el uso comercial-servicios.

Figura 2. Distribución de consumos según usos y superficies catastrales según usos

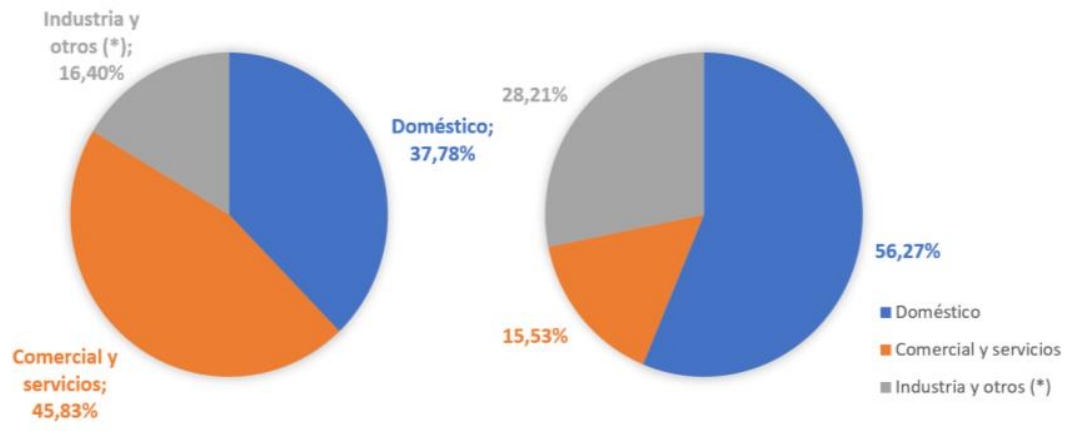

Fuente: Elaboración propia con datos del Ayuntamiento de Barcelona. (2014).

Tablas 2 y 3. Distribución de consumos según usos y superficies catastrales según usos

\begin{tabular}{|l|r|r|}
\hline $\begin{array}{r}\text { DISTRIBUCION DE CONSUMO DE ENERGIA FINAL POR SECTORES } \\
\text { sector }\end{array}$ & $\begin{array}{r}\text { Consumo final } \\
\text { (GWh) }\end{array}$ & $\begin{array}{r}\text { Distribución por uso } \\
\text { (\%) }\end{array}$ \\
\hline Doméstico & $4.393,03$ & $37,78 \%$ \\
Comercial y servicios & $5.329,15$ & $\mathbf{4 5 , 8 3 \%}$ \\
Industria y otros (*) & $1.906,62$ & $16,40 \%$ \\
\hline TOTAL & $\mathbf{1 1 . 6 2 8 , 7 9}$ & $\mathbf{1 0 0 , 0 0 \%}$ \\
\hline
\end{tabular}

\begin{tabular}{|l|rr|}
\hline \multicolumn{1}{|c|}{ SUPERFICIES DE USOS SEGÚN CATASTRO, 2014 } & Superficie construida \\
Sector & $\mathbf{m t 2}$ & $\begin{array}{c}\text { Distribución por uso } \\
\%\end{array}$ \\
\hline Doméstico & 63.960 .051 & $\mathbf{5 6 , 2 7 \%}$ \\
Comercial y servicios & 17.648 .634 & $\mathbf{1 5 , 5 3 \%}$ \\
Industria y otros $\left(^{*}\right)$ & 32.061 .658 & $\mathbf{2 8 , 2 1 \%}$ \\
TOTAL & $\mathbf{1 1 3 . 6 7 0 . 3 4 3}$ & $\mathbf{1 0 0 , 0 0 \%}$ \\
\hline
\end{tabular}

Fuente: Elaboración propia con datos del Ayuntamiento de Barcelona. (2014).

Para el caso del uso habitacional esta relación no es tan clara. El consumo energético más bien se asocia a la renta familiar, la densidad habitacional y/o las tipologías habitacionales.

Tomando en cuenta lo anterior se propone como metodología:

- 1 Comprobar si existe una correlación lineal entre la superficie per cápita comercialservicios (sup/mt2) y el consumo per cápita por distrito.

- $2^{\text {a }}$ Si la correlación es significativa, generar un modelo basado entre estos dos indicadores, de forma de distribuir el consumo per cápita a nivel de barrios.

- $\quad 3$ o Desagregar el consumo domiciliario del total de manera de evaluar los resultados.

Si distribuimos la superficie de usos "comercial y servicios", por los habitantes totales del barrio, obtendremos un índice que llamaremos "superficie comercial per cápita" (m2/hab).

Ahora comprobaremos qué grado de relación tiene el consumo (1) y la superficie comercial (2), para esto ocuparemos el coeficiente de correlación de Pearson. Como vemos en el gráfico y el cuadro 5, existe una alta correlación entre los campos comparados, pero los datos de Ciutat Vella, distorsionan los resultados. Para obtener un modelo más preciso se excluirán los datos de este distrito. 


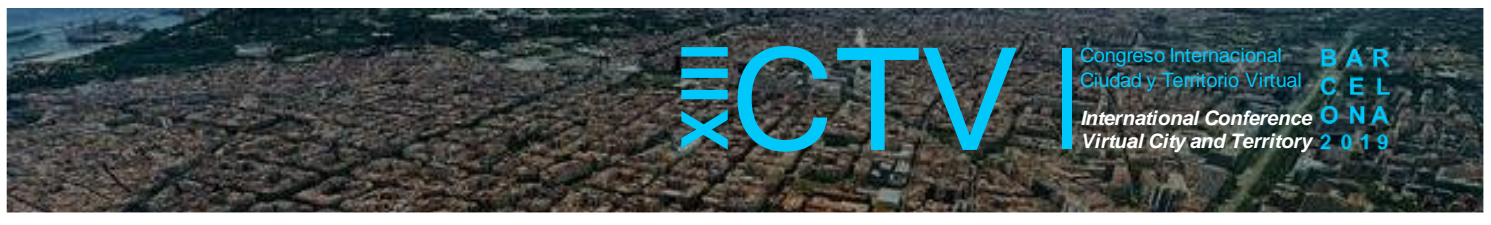

Tabla 4. Comparación consumo energético per cápita - superficie comercial per cápita

\begin{tabular}{|c|c|c|c|c|c|}
\hline & & & 1 & & 2 \\
\hline DISTRITO & $\begin{array}{c}\text { № Hab (1) } \\
\text { hab }\end{array}$ & $\begin{array}{l}\text { Ingreso familiar (2) } \\
\text { indice RFD. Barc. }=100\end{array}$ & $\begin{array}{c}\text { Consumo percápita (3) } \\
\text { MWh/hab }\end{array}$ & $\begin{array}{c}\text { SUPERFICIE COMERCIAL SERVICIOS } \\
\text { MT2 }\end{array}$ & $\begin{array}{l}\text { SUPERFICIE COMERCIAL per cápita } \\
\text { MT2/HAB }\end{array}$ \\
\hline Ciutat vella & 100.685 & 79,7 & 8,89 & 2.122 .664 & 21,08 \\
\hline Eixample & 263.565 & 115,9 & 9,15 & 4.712 .729 & 17,88 \\
\hline Sants-Montjüic & 180.824 & 75,8 & 8,18 & 2.233 .005 & 12,35 \\
\hline Les corts & 81.200 & 139,7 & 9,52 & 1.349 .472 & 16,62 \\
\hline Sarriá Sant Gervasi & 145.761 & 184,3 & 8,06 & 1.745 .037 & 11,97 \\
\hline Grácia & 120.273 & 108,5 & 6,01 & 812.417 & 6,75 \\
\hline Horta-Guinardó & 166.950 & 77,7 & 6,01 & 650.249 & 3,89 \\
\hline Nou Barris & 164.516 & 53,7 & 3,72 & 674.687 & 4,10 \\
\hline Sant Andreu & 164.516 & 73 & 6,1 & 976.425 & 5,94 \\
\hline Sant Martí & 232.629 & 85,6 & 8,08 & 2.371 .949 & 10,20 \\
\hline
\end{tabular}

Fuente: Elaboración propia desde datos del departamento de estadística y difusión de datos del Ayuntamiento de Barcelona. (2014).

Figura 3. Comprobación de correlación entre consumo per cápita y superficie comercial per cápita
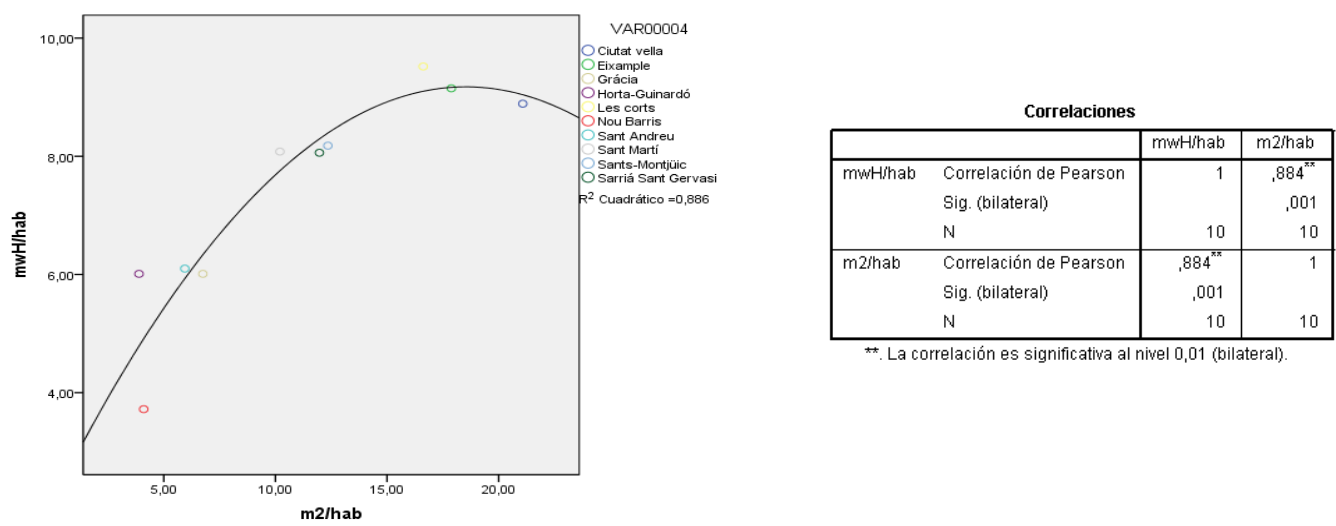

Fuente: Elaboración propia desde datos del departamento de estadística y difusión de datos del Ayuntamiento de Barcelona. (2014).

Desde los datos ya correlacionados, generamos un modelo de regresión lineal expresada en la función: $y=3,903+0,331^{*} x$, donde $x$ es la superficie comercial per cápita. Con lo anterior obtendremos el consumo per cápita de los barrios desde los cuales podremos calcular el consumo de $\mathrm{CO} 2$.

Figura 4. Modelo de regresión lineal entre consumo per cápita y superficie comercial per cápita
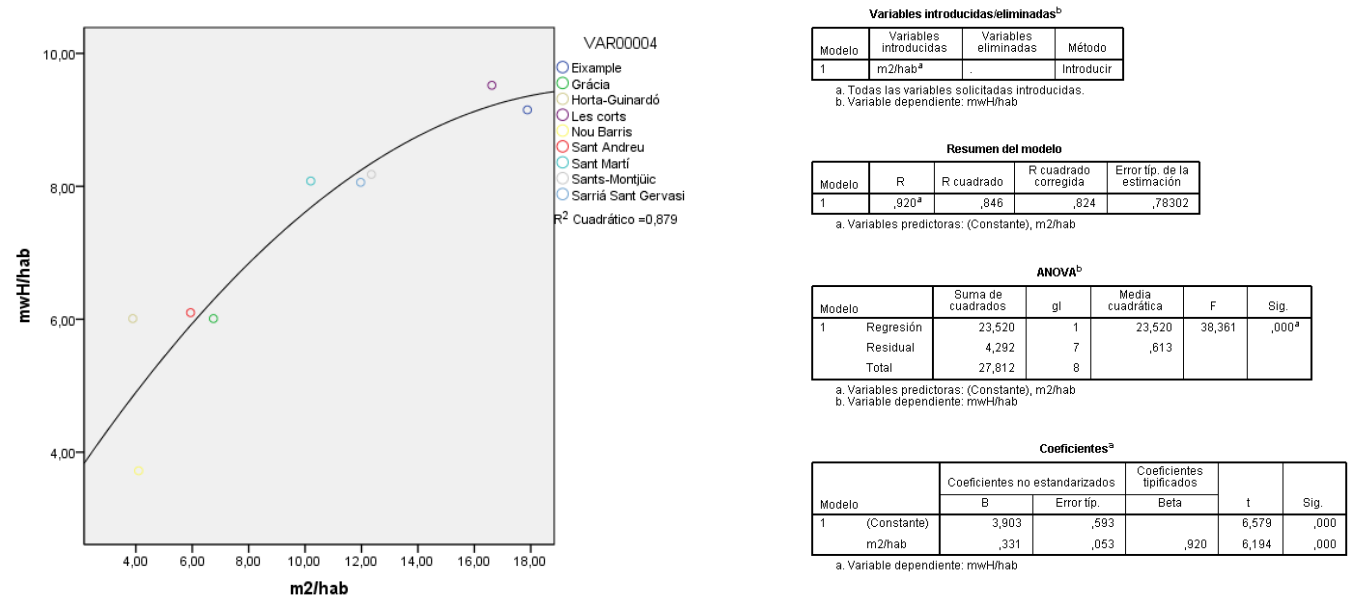

Fuente: Elaboración propia con datos del Ayuntamiento de Barcelona. (2014). 


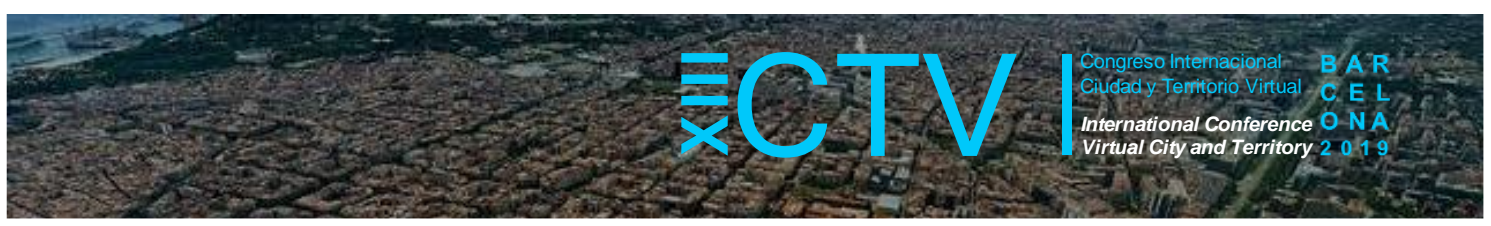

Como comprobación de los cálculos podemos comparar el resultado del consumo total del distrito dado por el modelo (1.114,64 GWh) (1), con el consumo total per cápita del distrito de la tabla 1, del balance de energía 2014. (1,174,83 GWh) (2). Ambas cifras son coherentes en cuanto a sus magnitudes.

Tabla 4. Comprobación del modelo de regresión

\begin{tabular}{|l|r|r|r|}
\hline \multicolumn{1}{|c}{ DISTRITO } & $\begin{array}{c}\text { No Hab (1) } \\
\text { hab }\end{array}$ & $\begin{array}{r}\text { Consumo percápita (2) } \\
\text { mwH/hab }\end{array}$ & \multicolumn{1}{c|}{$\begin{array}{c}\text { Consumo total } \\
\text { GwH }\end{array}$} \\
\hline Ciutat vella & 100.685 & 8,89 & 895,09 \\
Eixample & 263.565 & 9,15 & $2.411,62$ \\
\hline Sants-Montjüic & 180.824 & 8,18 & $1.479,14$ \\
Les Corts & 81.200 & 9,52 & 773,02 \\
Sarriá Sant Gervasi & 145.761 & 8,06 & $\mathbf{1 . 1 7 4 , 8 3}$ \\
Grácia & 120.273 & 6,01 & 722,84 \\
\hline Horta-Guinardó & 166.950 & 6,01 & $1.003,37$ \\
\hline Nou Barris & 164.516 & 3,72 & 612,00 \\
\hline Sant Andreu & 164.516 & 6,1 & $1.003,55$ \\
\hline Sant Martí & 232.629 & 8,08 & $1.879,64$ \\
\hline
\end{tabular}

\begin{tabular}{|c|c|c|}
\hline BARRIO & Consumo per cápita & Consumo total \\
\hline & MWh & GWh \\
\hline Sant Gervasi - Galvany & 9,96 & 464,76 \\
\hline Vallvidrera, el Tibidabo i les Planes & 7,80 & 36,00 \\
\hline Sant Gervasi - la Bonanova & 6,85 & 173,81 \\
\hline les Tres Torres & 6,55 & 107,36 \\
\hline el Putxet i el Farró & 6,29 & 183,54 \\
\hline \multirow[t]{2}{*}{ Sarrià } & 6,04 & 149,16 \\
\hline & TOTAL & $1.114,64$ \\
\hline
\end{tabular}

1

Fuente: Elaboración propia.

\subsection{Cálculo del consumo energético domiciliario}

Para entender mejor los resultados de los consumos por barrios se desagregaron de estos, los consumos domiciliarios. Para esto volvemos a la relación entre consumo doméstico y superficie habitacional desde los datos de las tablas 1 y 2 , en donde el $56,27 \%$ de la superficie consume el $37,78 \%$ del total de GWh/año. Sin embargo, esta proporción es referida a toda la ciudad de Barcelona.

Una forma simplificada de escalar esta relación a los distritos, tomando en cuenta sus diferencias en cuanto a renta, densidad, tipologías de vivienda, etc., es vincular la densidad habitacional ( $\mathrm{m} 2$ vivienda/ hab.) al consumo por $\mathrm{mt} 2$. Para lograr esto primero se plantea un INDEX de densidad habitacional en donde 100 es igual a la densidad habitacional de Barcelona. Luego se genera una constante entre consumo y superficie para, finalmente, relacionar esto con el porcentaje de superficie habitacional de cada distrito. Lo anterior se resumen en las siguiente formula:

- $I D * 0,6714 * R 1{ }^{*} C P=$ Consumo doméstico per cápita (GWh).

- $\quad I D=$ Index de densidad habitacional

- CONSTANTE $=37,78 \% / 56,27 \%=0,6714$

- $\quad \mathrm{R} 1=$ INDICE porcentaje de superficie habitacional respecto al total catastral del

- $\mathrm{CP}=$ Consumo per cápita $(\mathrm{MWh} / \mathrm{hab})$. 


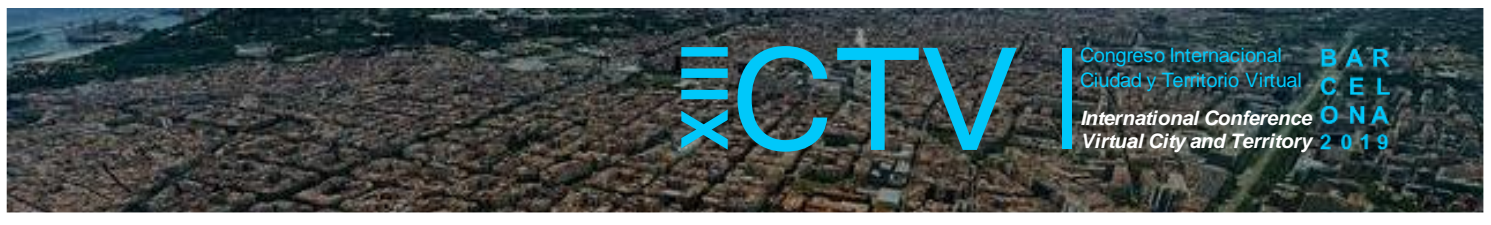

Tabla 5. Comparación entre RDF, consumo energético y densidad habitacional

\begin{tabular}{|c|c|c|c|c|c|c|c|}
\hline DISTRITO & $\begin{array}{c}\text { № } \mathrm{Hab}(1) \\
\text { hab }\end{array}$ & $\begin{array}{c}\text { Ingreso familiar (2) } \\
\text { indice RFD. Barc.=100 }\end{array}$ & $\begin{array}{c}\text { Consumo percápita (3) } \\
\text { MWh/hab }\end{array}$ & $\begin{array}{l}\text { Consumo total } \\
\text { MWh/hab }\end{array}$ & $\begin{array}{l}\text { Sup. Vivienda } \\
\mathrm{m} 2\end{array}$ & $\begin{array}{c}\text { Densidad habitacional } \\
\mathrm{m} 2 / \mathrm{hab}\end{array}$ & \begin{tabular}{|c|} 
Indice densidad base 100 \\
$100=$ Barcelona
\end{tabular} \\
\hline Ciutat vella & $100.685,00$ & 79,7 & 8,89 & $895.089,65$ & $3.960 .520,00$ & 39,34 & 99,69 \\
\hline Eixample & $263.565,00$ & 115,9 & 9,15 & $2.411 .619,75$ & $12.825 .413,00$ & 48,66 & 123,32 \\
\hline Sants-Montjüuic & $180.824,00$ & 75,8 & 8,18 & $1.479 .140,32$ & $6.053 .917,00$ & 33,48 & 84,85 \\
\hline Les corts & $81.200,00$ & 139,7 & 9,52 & $773.024,00$ & $3.716 .596,00$ & 45,77 & 116,00 \\
\hline Sarriá Sant Gervasi & $145.761,00$ & 184,3 & 8,06 & $1.174 .833,66$ & $8.409 .786,00$ & 57,70 & 146,22 \\
\hline Grácia & $120.273,00$ & 108,5 & 6,01 & $722.840,73$ & $5.160 .354,00$ & 42,91 & 108,73 \\
\hline Horta-Guinardó & $166.950,00$ & 77,7 & 6,01 & $1.003 .369,50$ & $5.753 .454,00$ & 34,46 & 87,34 \\
\hline Nou Barris & $164.516,00$ & 53,7 & 3,72 & $611.999,52$ & $5.008 .333,00$ & 30,44 & 77,15 \\
\hline Sant Andreu & $164.516,00$ & 73 & 6,1 & $1.003 .547,60$ & $4.921 .928,00$ & 29,92 & 75,82 \\
\hline Sant Martí & $232.629,00$ & 85,6 & 8,08 & $1.879 .642,32$ & $8.149 .750,00$ & 35,03 & 88,78 \\
\hline TOTAL & $1.620 .919,00$ & & & & $63.960 .051,00$ & 39,46 & \\
\hline
\end{tabular}

Fuente: Elaboración propia con datos del Ayuntamiento de Barcelona. (2014).

A partir de los datos del consumo doméstico habitacional se generó un modelo basado en el índice de ingreso familiar, con el cual se obtiene los consumos por barrios, teniendo como hipótesis la directa relación entre consumo energético y renta.

Tabla 6. Correlación y modelo de regresión entre consumo domiciliar y renta

\begin{tabular}{|l|l|r|r|r|}
\multicolumn{7}{c|}{ Resumen del modelo } \\
\hline Modelo & $\mathrm{R}$ & R cuadrado & $\begin{array}{c}\text { R cuadrado } \\
\text { corregida }\end{array}$ & $\begin{array}{c}\text { Error típ. de la } \\
\text { estimación }\end{array}$ \\
\hline 1 & $.894^{\mathrm{a}}$ &, 800 & .774 &, 52574 \\
\hline
\end{tabular}

a. Variables predictoras: (Constante), ingreso

\begin{tabular}{|c|c|c|c|c|c|c|}
\hline \multicolumn{7}{|c|}{ ANOVA $^{\mathrm{b}}$} \\
\hline & & $\begin{array}{c}\text { Sumade } \\
\text { cuadrados }\end{array}$ & gl & $\begin{array}{c}\text { Media } \\
\text { cuadrática }\end{array}$ & $\mathrm{F}$ & Sig. \\
\hline \multirow[t]{3}{*}{1} & Regresión & 8,820 & 1 & 8,820 & 31,908 & $.000^{\mathrm{a}}$ \\
\hline & Residual & 2,211 & 8 & .276 & & \\
\hline & Total & 11,031 & 9 & & & \\
\hline
\end{tabular}

a. Variables predictoras: (Constante), ingreso
b. Variable dependiente: consumo domestico

Fuente: Elaboración propia.

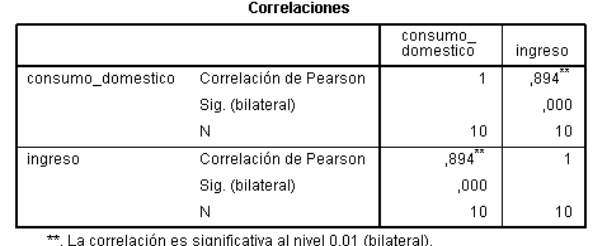

*. La correlación es signific ativa al nivel 0,01 (bilateral).

\subsection{Cálculo de la entropía informativa $(H)$.}

En cuanto al índice $\mathrm{H}$, se calculó aplicando la fórmula de Shannon, aplicada a los siguientes datos, obtenidos desde la página web del departamento de estadísticas del ayuntamiento de Barcelona y la sede electrónica del Catastro, todos relacionados con la diversidad de cada barrio:

- Inventario de locales comerciales en $1^{\circ}$ piso.

- Locales catastrales según uso.

- Nivel académico de la población

- Nacionalidad de la población.

Como ejemplo de la metodología aplicada, se expone a continuación el cálculo de $(\mathrm{H})$, respecto a los usos de los locales catastrados, excluyendo el uso de habitacional.

En este caso desde el catastro se definen 9 usos por cada barrio. El objetivo es medir primero la probabilidad de cada uso respecto al total de locales por barrio para luego aplicar la fórmula de Shannon que en este caso sería LOG $(\mathrm{Pi}, 2){ }^{*} \mathrm{Pi}$, donde $\mathrm{Pi}$ es la probabilidad. La sumatoria por barrio de estas magnitudes expresa la cantidad de información en bits producida. 


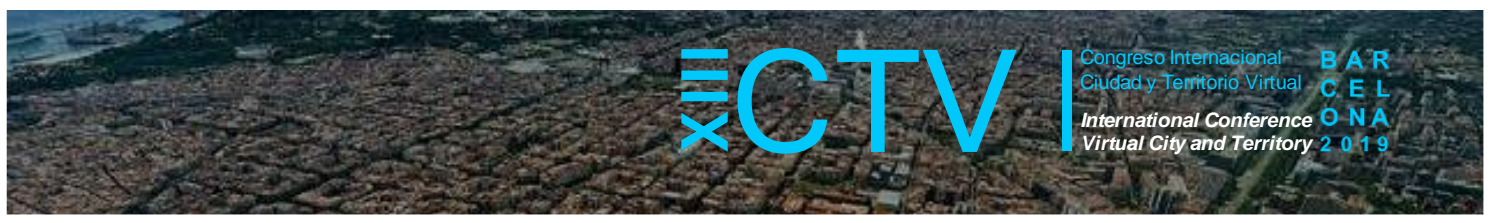

Para obtener el cálculo del total de la información por barrio respecto a las 4 variables desarrolladas, se realiza una suma ponderada por un factor de relevancia. Se estima así, por ejemplo, que las tipologías de usos y el inventario de locales comerciales tienen más relevancia en cuanto a la estimación del grado de complejidad del barrio, que el nivel académico de la población o la Nacionalidad de esta.

Tabla 7. Tipologías de usos, excluyendo la vivienda

\begin{tabular}{|c|c|c|c|c|c|c|c|c|c|c|c|}
\hline \multicolumn{11}{|c|}{ TIPOLOGIA DE LOCALES CATASTRALES, USOS DESAGREGADOS EXCLUIDA LA VIVIENDA(2017) } & \\
\hline Barrios & $\begin{array}{c}\text { TOTAL LOCALES } \\
\text { un }\end{array}$ & $\begin{array}{c}\text { Comercio } \\
\text { un }\end{array}$ & $\begin{array}{c}\text { Industria } \\
\text { un }\end{array}$ & $\begin{array}{c}\text { Oficinas } \\
\text { un }\end{array}$ & $\begin{array}{c}\text { Educación } \\
\text { un }\end{array}$ & $\begin{array}{c}\text { Sanidad } \\
\text { un }\end{array}$ & $\begin{array}{c}\text { Hosteleria } \\
\text { un }\end{array}$ & $\begin{array}{c}\text { Deportivo } \\
\text { un }\end{array}$ & $\begin{array}{c}\text { Religioso } \\
\text { un }\end{array}$ & $\begin{array}{c}\text { Espectáculos } \\
\text { un }\end{array}$ & \\
\hline Vallvidrera, el Tibidabo i les Planes & 842,00 & 160,00 & 366,00 & 14,00 & 59,00 & 50,00 & 27,00 & 117,00 & 37,00 & 12,00 & \\
\hline Sarrià & $3.983,00$ & $1.219,00$ & $1.088,00$ & 297,00 & 368,00 & 193,00 & 99,00 & 589,00 & 129,00 & 1,00 & \\
\hline les Tres Torres & $2.341,00$ & 482,00 & $1.042,00$ & 359,00 & 70,00 & 276,00 & 28,00 & 39,00 & 44,00 & 1,00 & \\
\hline Sant Gervasi - la Bonanova & $3.993,00$ & $1.217,00$ & 874,00 & 533,00 & 437,00 & 448,00 & 135,00 & 213,00 & 120,00 & 16,00 & \\
\hline Sant Gervasi - Galvany & $11.049,00$ & $3.893,00$ & $2.646,00$ & $3.225,00$ & 302,00 & 330,00 & 357,00 & 87,00 & 135,00 & 74,00 & \\
\hline el Putxet $i$ el Farró & $3.857,00$ & $1.390,00$ & $1.360,00$ & 619,00 & 183,00 & 117,00 & 79,00 & 54,00 & 41,00 & 14,00 & \\
\hline \multicolumn{11}{|l|}{ PROBABILIDAD } & \\
\hline Vallvidrera, el Tibidabo i les Planes & & 0,190 & 0,435 & 0,017 & 0,070 & 0,059 & 0,032 & 0,139 & 0,044 & 0,014 & \\
\hline Sarrià & & 0,306 & 0,273 & 0,075 & 0,092 & 0,048 & 0,025 & 0,148 & 0,032 & 0,000 & \\
\hline les Tres Torres & & 0,206 & 0,445 & 0,153 & 0,030 & 0,118 & 0,012 & 0,017 & 0,019 & 0,000 & \\
\hline Sant Gervasi - la Bonanova & & 0,305 & 0,219 & 0,133 & 0,109 & 0,112 & 0,034 & 0,053 & 0,030 & 0,004 & \\
\hline Sant Gervasi - Galvany & & 0,352 & 0,239 & 0,292 & 0,027 & 0,030 & 0,032 & 0,008 & 0,012 & 0,007 & \\
\hline el Putxet i el Farró & & 0,360 & 0,353 & 0,160 & 0,047 & 0,030 & 0,020 & 0,014 & 0,011 & 0,004 & \\
\hline INFORMACION & & & & & & & & & & & TOTAL (Bits) \\
\hline Vallvidrera, el Tibidabo i les Planes & & 0,46 & 0,52 & 0,10 & 0,27 & 0,24 & 0,16 & 0,40 & 0,20 & 0,09 & 2,43 \\
\hline Sarrià & & 0,52 & 0,51 & 0,28 & 0,32 & 0,21 & 0,13 & 0,41 & 0,16 & 0,00 & 2,55 \\
\hline les Tres Torres & & 0,47 & 0,52 & 0,41 & 0,15 & 0,36 & 0,08 & 0,10 & 0,11 & 0,00 & 2,21 \\
\hline Sant Gervasi - la Bonanova & & 0,52 & 0,48 & 0,39 & 0,35 & 0,35 & 0,17 & 0,23 & 0,15 & 0,03 & 2,67 \\
\hline Sant Gervasi - Galvany & & 0,53 & 0,49 & 0,52 & 0,14 & 0,15 & 0,16 & 0,06 & 0,08 & 0,05 & 2,18 \\
\hline El Putxet i el Farró & & 0,53 & 0,53 & 0,42 & 0,21 & 0,15 & 0,11 & 0,09 & 0,07 & 0,03 & 2,15 \\
\hline
\end{tabular}

Fuente: Elaboración propia con datos del Ayuntamiento de Barcelona. (2014).

\section{Resultados}

\subsection{Entropía física (S)}

Respecto al cálculo del consumo de $\mathrm{CO} 2$, podemos decir que, en general, los consumos domiciliarios tienen menor variación entre barrios y se ubican todos en torno a la media de 5 $\mathrm{MWh} /$ hab. Al contrario, el uso comercial genera una marcada variación comparativa en el consumo. Como ejemplo, el barrio Tres Torres, es el que tiene mayores rentas familiares, pero, al contrario, tiene la menor superficie de uso comercial (solo $8.7 \%$ del total catastral). Esto hace que se encuentre en el $4^{a}$ posición de consumo. El $30 \%$ de la superficie construida del barrio Sant Gervasi - Galvany corresponde al sector comercial y servicios, en contraste con el barrio de Sarrià en donde sólo el 16\% corresponde a este uso. Estos extremos se explicarían por la relación comercio/consumo antes descrita.

\section{Figura 7. Gráficos de consumo de $\mathrm{CO} 2$ per capitas desagregados}
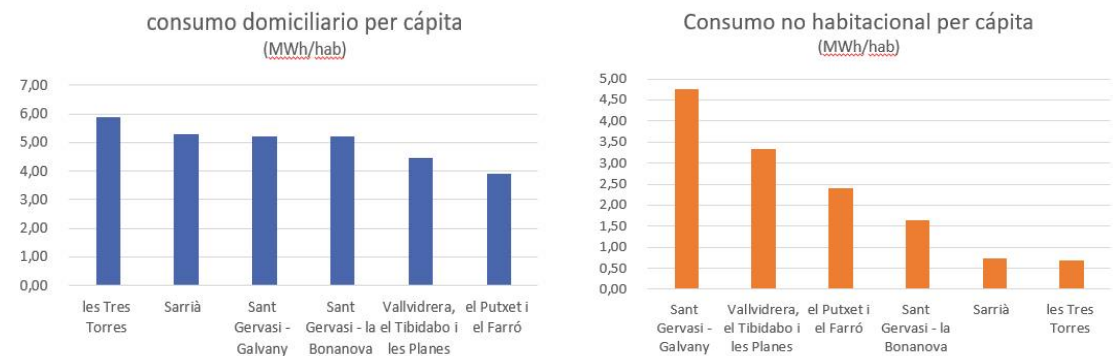

Fuente: Elaboración propia. 
Figura 8. Consumo total de $\mathrm{CO} 2$ per cápita por barrio

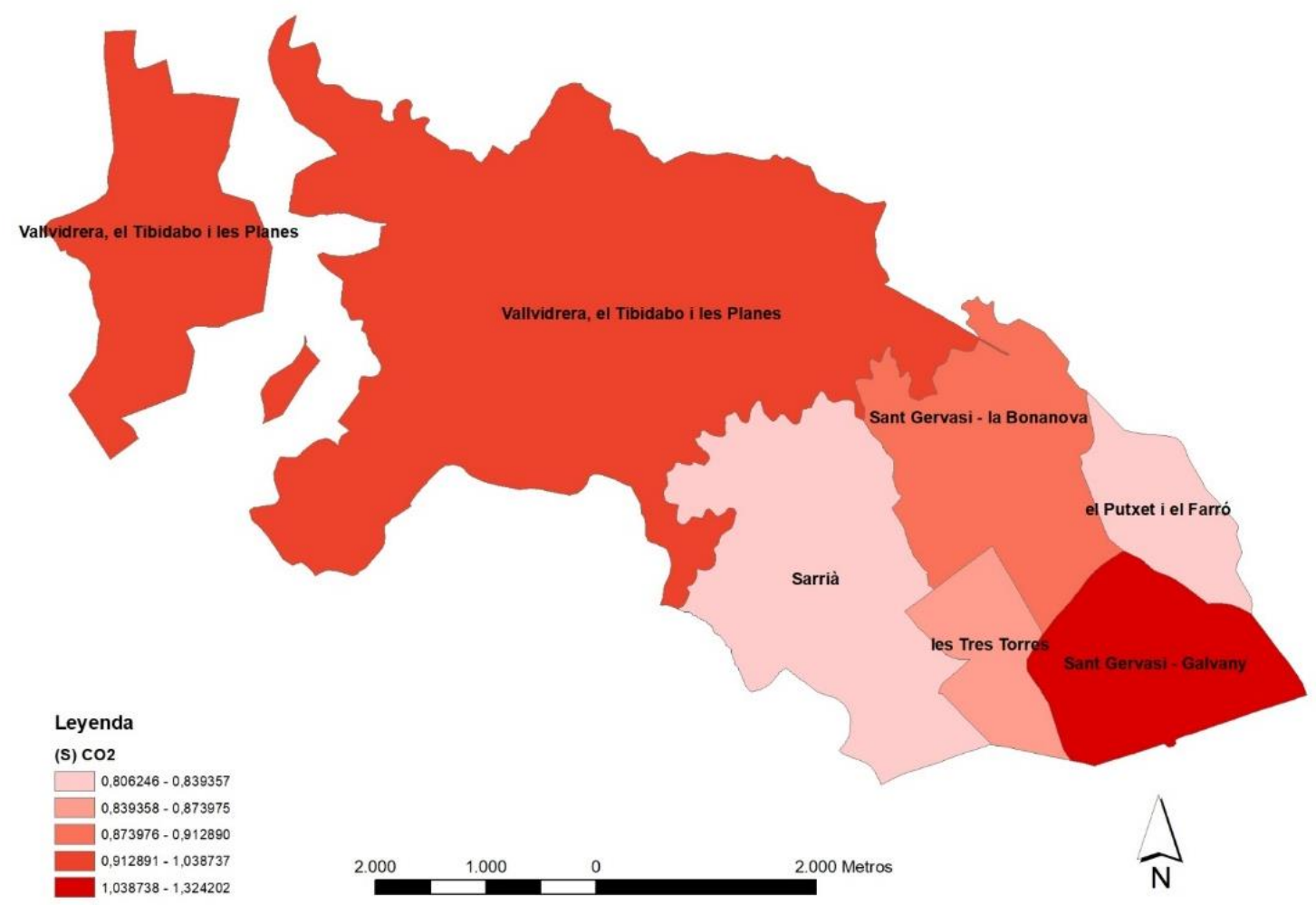

Fuente: Elaboración propia.

Figura 9. Consumo total de CO2 per cápita por barrio. (MWh/hab)

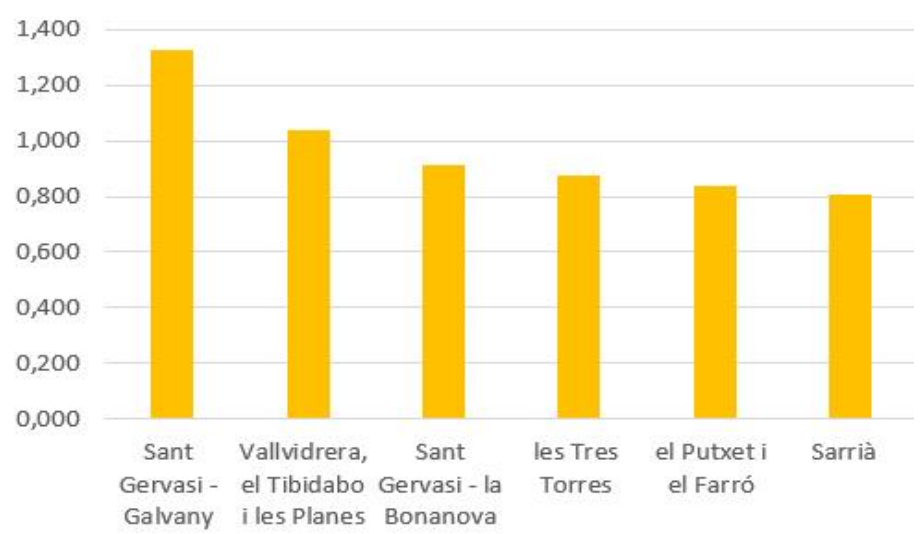

Fuente: Elaboración propia.

\subsection{Entropía informativa $(H)$.}

En cuanto al cálculo de la información, podemos ver que el barrio más complejo es el de Sant Gervasi - la Bonanova, principalmente debido a su mixtura de usos y la relación de estos con el uso habitacional. Por el contrario, les tres torres, es el barrio que produce menor información y por lo tanto es el que menos mitiga sus emisiones de $\mathrm{CO} 2$. 
Tabla 8. Producción de información por barrio. (bits)

\begin{tabular}{|c|c|c|c|c|c|}
\hline INFORMACION & Nacionalidades & Niv. Educacional & Tipos catastrales & Act.comerciales & PROMEDIO pond. \\
\hline & bits & bits & bits & bits & (H) \\
\hline Vallvidrera, el Tibidabo i les Planes & 1,240 & 1,800 & 2,427 & 3,323 & 2,16 \\
\hline Sarrià & 1,173 & 1,661 & 2,546 & 3,644 & 2,26 \\
\hline les Tres Torres & 0,909 & 1,549 & 2,206 & 3,514 & 2,07 \\
\hline Sant Gervasi - la Bonanova & 1,079 & 1,625 & 2,668 & 3,713 & 2,30 \\
\hline Sant Gervasi - Galvany & 1,158 & 1,596 & 2,177 & 3,576 & 2,12 \\
\hline El Putxet i el Farró & 1,223 & 1,647 & 2,146 & 3,580 & 2,13 \\
\hline FACTOR DE PONDERACIÓN & 1,000 & 1,000 & 2,000 & 2,000 & \\
\hline
\end{tabular}

Fuente: Elaboración propia.

Figura 10. Producción de información por barrio. (bits)

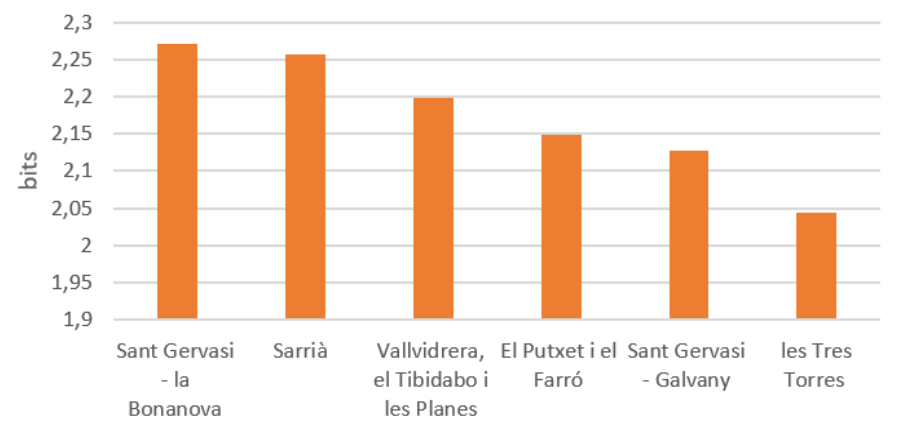

Fuente: Elaboración propia.

\subsection{Ecoeficiencia $(H / S)$}

Para calcular el índice de ecoeficiencia, se aplica la formula $\mathrm{H} / \mathrm{S}$, la cual divide los resultados de la producción anual de $\mathrm{CO} 2$ por barrio, por el promedio ponderado de los cálculos de complejidad urbana para cada uno los 4 índices analizados. Los resultados principales se pueden expresar en lo siguiente:

- El barrio de Sant Gervasi-Galvany obtuvo un índice S de 1.32 tCO2eq, un índice H de 2.127 bits y un índice de ecoeficiencia de 1.606 tCO2eq/bits.

- En cuanto al barrio de Sarriá, este obtuvo un índice S de 0.81 tCO2eq, un índice H de 2.256 bits y un índice de Ecoeficiencia de 2.798 tCO2eq/bits.

Tabla 9. Índice de ecoeficiencia por barrios del distrito Sarriá Sant Gervasi

\begin{tabular}{|c|c|c|c|}
\hline ECOEFICIENCIA & ENTROPIA INFORMATIVA & ENTROPIA FÍSICA & INDICE ECOEFICIENCIA \\
\hline & (H) & (S) & $(\mathrm{H} / \mathrm{S})$ \\
\hline 2. Sarrià & 2,256 & 0,81 & 2,798 \\
\hline 6. El Putxet i el Farró & 2,149 & 0,84 & 2,560 \\
\hline 4. Sant Gervasi - la Bonanova & 2,271 & 0,91 & 2,488 \\
\hline 3. les Tres Torres & 2,045 & 0,87 & 2,339 \\
\hline 1. Vallvidrera, el Tibidabo i les Planes & 2,197 & 1,04 & 2,115 \\
\hline 5. Sant Gervasi - Galvany & 2,127 & 1,32 & 1,606 \\
\hline
\end{tabular}

Fuente: Elaboración propia. 


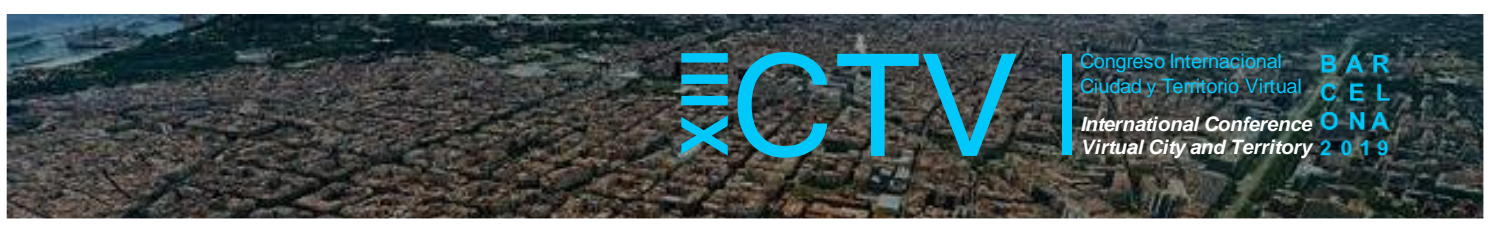

Figura 11. Índice de ecoeficiencia por barrios del distrito Sarriá Sant Gervasi

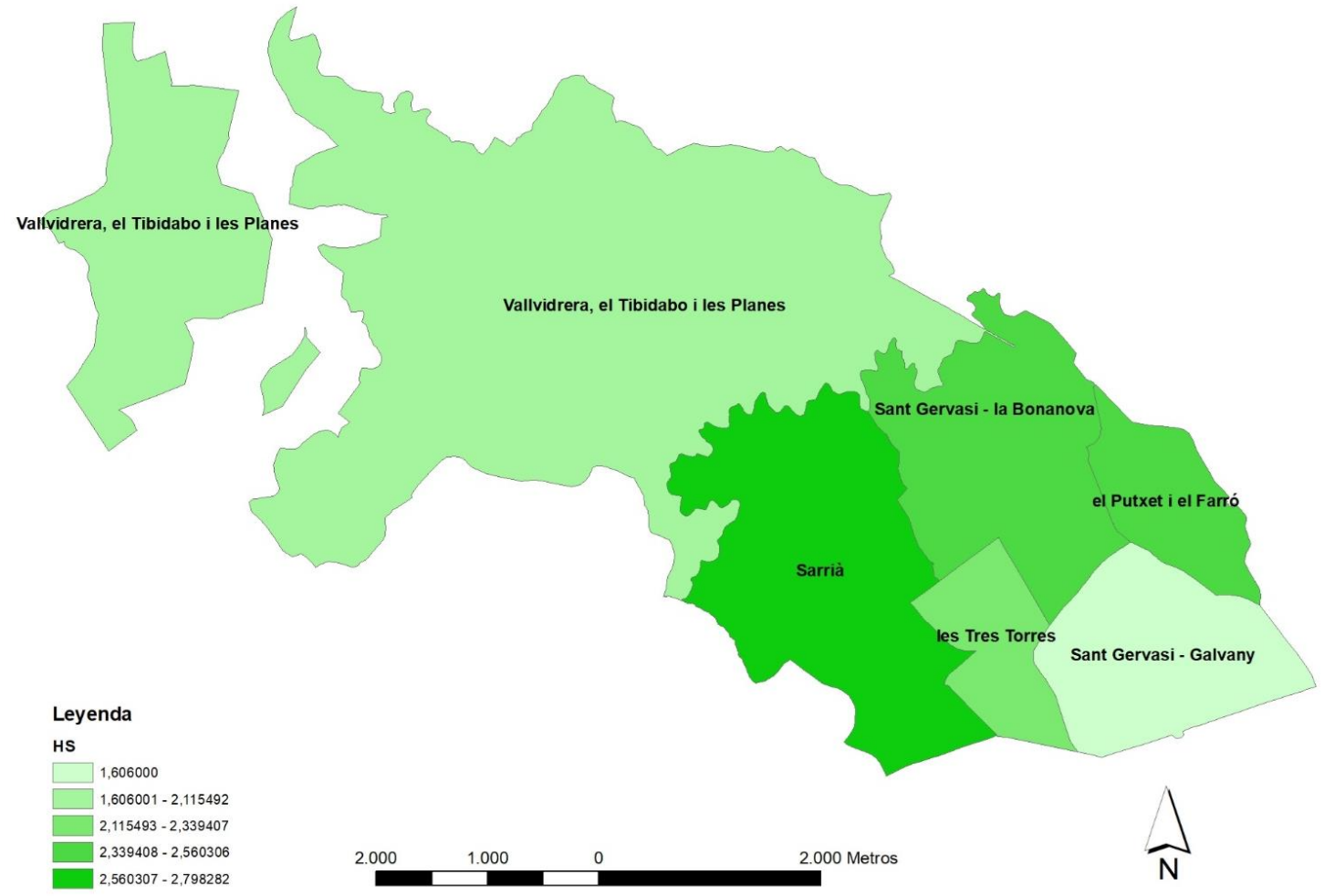

Fuente: Elaboración propia.

\section{Conclusiones}

El barrio que más entropía física obtuvo, Sant Gervasi - Galvany, es el menos ecoeficiente; la información que produce no compensa las altas emisiones de $\mathrm{CO} 2$ que el sector comercial y de servicios genera. De los resultados podemos deducir que, en general, los consumos domiciliarios tienen menor variación entre barrios y se ubican todos en torno a la media de 5 $\mathrm{MWh} / \mathrm{hab}$. Al contrario, el uso comercial genera una marcada variación comparativa en el consumo.

De lo anterior se puede interpretar, que la gran cantidad de información generada por modelos de ciudad compacta, como es el caso de ciertas áreas de Barcelona, no necesariamente compensa la gran producción de "huella ecológica".

Este tipo de análisis comparativo de barrios tienen el potencial de generar información a nivel municipal, con la cual poder proyectar acciones de mitigación desde la planificación, a una escala espacial acotada y específica, desde la cual es más fácil poder generar medidas simples, directas y consensuadas con la comunidad.

Como ejemplo, en el caso específico de este estudio, por el lado de los incentivos, se podría pensar en una especie de "bono verde local", enfocado a las edificaciones comerciales y de servicio, que premiaran la implementación de sistemas de generación de energía sustentable, a través de descuentos en impuestos territoriales y/o alguna clase de certificación ecológica local. 


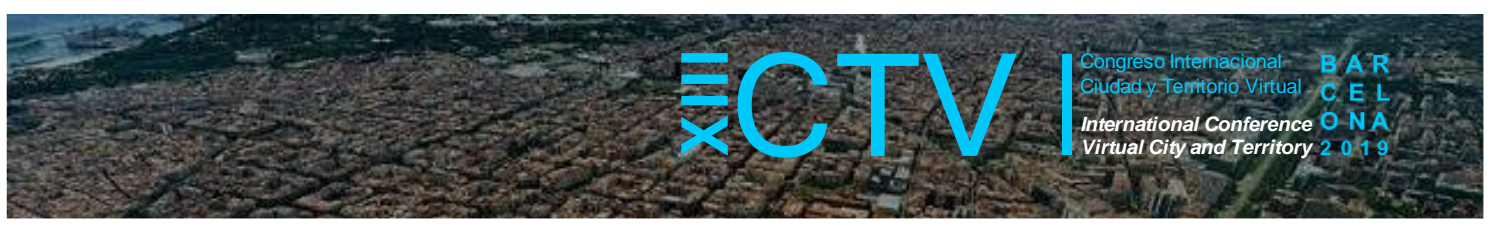

Contribuciones de los autores: El primer autor ha desarrollado el artículo en base a un ejercicio académico en el marco del Master Planificación urbana y sostenibilidad de la universidad Politécnica de Catalunya, el segundo autor es el catedrático que formulo el ejercicio desde el ejercicio desde el cual se desarrolla este trabajo

Conflicto de Intereses: Los autores declaran que no hay conflicto de intereses.

\section{Bibliografía}

Declaración del Milenio. (2000). Resolución aprobada por la asamblea general de las Naciones Unidas. Recuperado de https://www.un.org/spanish/milenio/ares552.pdf.

Cambio Climático 2014 mitigación del cambio climático, resumen para responsables de políticas. (2014). IPCC Grupo intergubernamental de expertos sobre el cambio climático. Recuperado de https://www.ipcc.ch/site/assets/uploads/2018/03/WG3AR5_SPM_brochure_es1.pdf

Garcia, V. M. y Fairén, L. V. (1980). Estructuras disipativas, algunas nociones básicas. Revista El Basilisco, (7), 08-13. https://dialnet.unirioja.es/servlet/articulo?codigo=2471729.

Wolman, A. (1965). The metabolism of cities. Scientific American. Recuperado de 213. 179-90. https://www.researchgate.net/publication/9244776_The_Metabolism_of_Cities.

Odum, T. (1983). Systems Ecology. Usa. Wiley.

Fariña, T. J. y Sánchez, J. R. (2002). Orden, desorden y entropía en la construcción de la ciudad. Revista del Departamento de Urbanística y Ordenación del Territorio, (7), 08-15. Recuperado de http://polired.upm.es/index.php/urban/article/view/339.

Molini, F. y Salgado, M. (2010). Superficie artificial y viviendas unifamiliares en España, dentro del debate entre ciudad compacta y ciudad difusa.

https://alojamientos.uva.es/guia_docente/uploads/2013/474/46059/1/Documento38.pdf

Wackernagel, M. y Rees, W. (1995). Our Ecological Footprint: Reducing Impact on the Earth. ISBN 0-86571-312-x.

Balance de la Energía. (2014). Ayuntamiento de Barcelona. Recuperado de http://energia.barcelona/sites/default/files/documents/balanc_energia_2014-r.pdf. 